\title{
DESIGN AND EVALUATION OF AN INTEGRATED AVIONICS ALERTING SYSTEM
}

\author{
Michael C. Dorneich, Patricia May Ververs, Michael D. Good \\ Honeywell Laboratories, Minneapolis, MN
}

\begin{abstract}
While today's aircraft have integrated alerting systems for conditions inside the aircraft (ECAM, EICAS), there is no comparable fully integrated alerting system for conditions outside of the aircraft. Current and near-future, separate alerting systems warn of conditions such as time-critical terrain, traffic, wind shear, clear air turbulence, wake vortices, each using different alerting and display philosophies to present information to the flight crew. Separate systems contribute to alert proliferation and the potential for multiple conflicting alerts during emergency situations. An integrated alerting system should deconflict alerts, and present information in an integrated fashion. In response to these issues, we have developed the Alerting and Notification of Conditions Outside the Aircraft (ANCOA) concept. Key aspects of the concept include: (1) deconflicting currently separate alerts such as TCAS and EGPWS; (2) categorization (weather, traffic, ground) and prioritization (time-critical, tactical and strategic) of alerts to reduce pilot information processing requirements; (3) directional, multidimensional aural cueing to allow quick "pre-processing" of the condition (this aids in time-critical responses and prioritizing the alerted condition relative to the ongoing task); and (4) integrated graphic presentation of conditions external to the aircraft to support better situation awareness. This paper discusses the theory and implementation of the ANCOA concept, and presents a usability study to evaluate the display configuration, ease-of-use, functionality, and navigation of information within an initial simulator prototype. Nine pilots participated in six scenarios under various conditions in the Honeywell Laboratories Medium-Fidelity Flight Simulator. Additionally, pilots conducted an information categorization task, filled out pre- and post-questionnaires, and were interviewed for qualitative assessments. The data were used to
\end{abstract}

assess if the ANCOA integrated system could effectively prioritize and de-conflict information, support improved detection and identification of threats, increase overall situation awareness, and support better planning decisions. A revised prototype is presented, based on the results of the evaluation.

\section{Introduction}

ANCOA was developed as a means to present alerting and notification information of conditions external to the aircraft. TCAS and EGPWS are two examples of separate systems and are aimed at very specific external conditions. There are two obvious voids in today's aircraft alerting system, both specific to conditions outside the aircraft: (1) the lack of integration among external condition alerting systems, and (2) the lack of strategic information to provide pilots with the resources and time to prepare for or avoid emerging situations.

The lack of integration among external condition alerting systems gives rise to two issues: (1) alert proliferation and (2) conflicting and contradictory information. Similar issues arose in the early 1970s, an emergency on the flight deck meant that the crew was inundated with visual/aural alerts attempting to direct their attention to the nature of the problem. On the L-1011, alone, almost 800 visual alerting functions were designed to inform the pilot of developing adverse conditions [1]. The introduction of integrated caution and warning systems onto the flight deck, such as Boeing's Engine Indicating and Crew Alerting System (EICAS) solved the alert proliferation problem for conditions inside the aircraft. However, a similar situation is arising from separate systems that alert the crew to conditions outside the aircraft. These alerting systems warn of time-critical terrain (e.g., EGPWS), traffic (e.g., TCAS), or various weather phenomena (e.g., reactive and predictive wind shear). Other independent alerting systems 
Michael C. Dorneich, Patricia M. Ververs, Michael D. Good (2001). "Design and Evaluation of an Integrated Avionics Alerting System", Proc. of the 20 $0^{\text {th }}$ Digital Avionics Systems Conference. Daytona Beach, FL, 14-18 October 2001

are under development, including clear air turbulence and wake vortices. ADS-B will extend the range of traffic alerts and various electronic communication links are beginning to allow much more information to reach the crew from ground sources, but with no integrated standard protocol for prioritizing and organizing the information to reduce the demands on pilot attention and information processing. Again, these alerting systems are separate, independent units manufactured by a variety of avionics suppliers, each using different alerting and display philosophies to present information to the crew.

The problem of conflicting or contradictory information is best described, in Figure 1, by an actual incident reported to NASA Aviation Safety and Reporting System (ASRS).

\begin{abstract}
Upon departure from LaGuardia on runway 13, whitestone climb, passing approximately $1000 \mathrm{ft}$ agl, a TCASIl traffic advisory (TA) 'traffic, traffic' sounded. At the time we were in instrument meteorological conditions. Immediately after that a resolution advisory (RA), 'reduce vertical speed,' came on with the TCASII target superimposed on our aircraft symbol. We began reducing our climb when an RA 'descend, descend' sounded with a vertical speed command of greater than $2000 \mathrm{fpm}$ annunciated. The target was still directly on top of us with its relative altitude displayed. We immediately commenced our descent and exited the clouds at $900 \mathrm{ft}$ agl at which time a GPWS warning came on saying 'too low, terrain.' By this time speed had built up to 280 knots. I then decided it was better to take a chance on hitting another airplane versus the sure thing of colliding with the ground, and directed the first officer to resume the climb and departure while I turned the transponder to TA only. At this time ATC gave us a new heading and altitude and I reported the TCASII RA maneuver. All this time either a TA, RA or GPWS warning had been going on and for a while 'descend, descend' and 'too low, terrain' were being broadcast simultaneously. The cockpit indeed was a cacophony of bells, whistles and flashing lights. (abbreviations were spelled out and nonessential text was added for readable; ASRS ACCESSION NUMBER: 403254)
\end{abstract}

Figure 1. ASRS incident report.

As described in the incident, the crew was receiving valid but opposing alerts from the TCAS and GPWS systems. In this time-critical, stressful situation, the pilots had to decide on their own which alert would take precedence and the appropriate action to take. Indeed this decision was made even more difficult by the blaring bells and whistles. Each system was designed with its own goals and objectives. Since the systems are separate and independent they do not have a common framework to share intent. Without a means to integrate and prioritize information, pilots are left on their own to de-conflict the alerts.
The second void identified with today's aircraft alerting systems was the lack of integrated strategic information for predictive situation awareness and planning ahead. The authors reasoned that if the crew is adequately informed of developing conditions many potential cautions and warnings can be avoided all together. Noticeably absent among the abundance of information provided on the flight deck is the availability of strategic information for look-ahead prediction, planning, and situation awareness. Though pilots are briefed about an hour prior to their planned departure concerning forecasts, advisories, and expected delays, in a dynamic environment, conditions are continually changing. Pilots generally have access to real-time tactical weather information from their weather radar systems and by looking out the windscreen. For up-to-date strategic information, pilots rely on supplemental information from the Automated Flight Service Station (AFSS) briefing facilities, communications with ATC, dispatch, and the 'party line,' by which pilots overhear other pilots reporting current conditions [2]. The only problem is that the information is presented verbally or textually and therefore needs to be processed, integrated, and visualized by the crew to understand the relevance to their current flight plan.

In response to these issues, we have developed the Alerting and Notification of Conditions Outside the Aircraft (ANCOA) concept. This concept is based on the application of human-centered guidelines and the development of a consistent philosophy for designing the components of an aural and visual alerting system. Key aspects of the concept are: (1) the ability to de-conflict currently separate alerts such as TCAS and EGPWS; (2) categorization (weather, traffic, ground, other) and prioritization (time-critical, tactical and strategic) of alerts to reduce pilot information processing requirements; (3) directional, multidimensional aural cueing to allow quick "pre-processing" of the condition (this aids in time-critical responses and in deciding the priority of the alerted condition relative to the on-going task); and (4) integrated graphic presentation of conditions external to the aircraft to support better situation awareness. ANCOA integrates information from independent alerting systems enabling multiple alerts of external conditions to be prioritized and de-conflicted before 
Michael C. Dorneich, Patricia M. Ververs, Michael D. Good (2001). "Design and Evaluation of an Integrated Avionics Alerting System", Proc. of the 20 $0^{\text {th }}$ Digital Avionics Systems Conference. Daytona Beach, FL, 14-18 October 2001

presentation to the crew. The goal was to support prompt, appropriate responses to adverse conditions based on good situation awareness.

\section{ANCOA}

The Alerting and Notification of Conditions Outside the Aircraft (ANCOA) concept proposed here creates an integrated alerting and notification system that assures that pilots are aware of, and informed about, environmental and other important conditions external to the aircraft. It integrates current alerting systems for external conditions, and provides integrated alerting for information transmitted from the ground, much of which is strategic in nature. Finally, it provides supporting information for external situation awareness in an integrated graphical format.

\section{ANCOA Philosophy}

ANCOA development followed humancentered design philosophy. This philosophy was applied to the look and feel of the alerting system as well as the processing requirements. Figure illustrates the functional requirements of ANCOA. These requirements are largely sequential in nature given an alerting situation. ANCOA utilizes aural and visual information to aid the pilot in performing each of these functions. The remainder of this section will describe issues related to each of the functional requirements of the ANCOA concept.

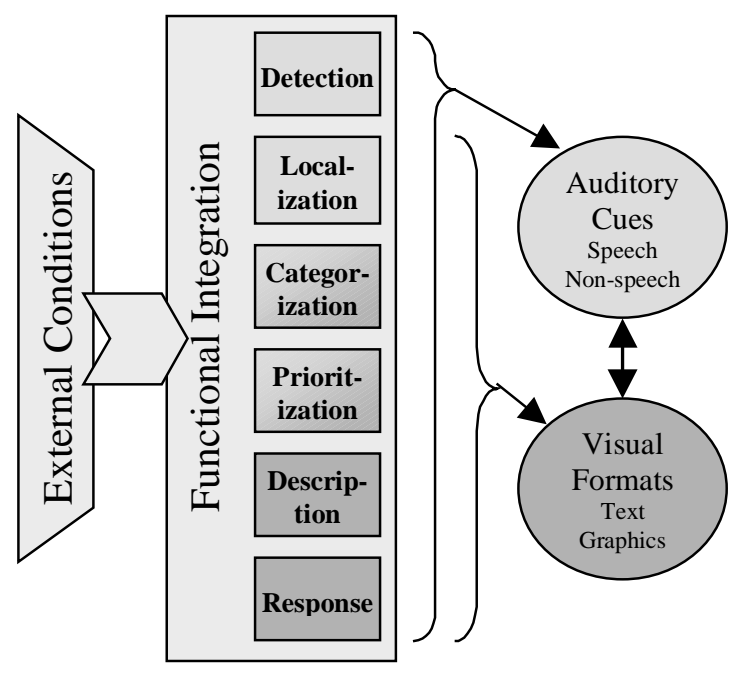

Figure 2. Functional Requirements
Detection. A pilot cannot respond to a situation that he or she does not know about, and so the detection by the pilot of anomalous conditions is the first responsibility of an effective alerting system. In the ANCOA concept, this function is best supported by an aural cue. Research has shown that the ability of an observer to detect a signal can be substantially improved if an aural warning is presented versus situations in which an aural warning is absent [3]. Aural information about the environment can be processed independently of the direction of the pilot's gaze. In the ANCOA concept, a second parameter, location, is used to aid in pilot detection of alerts. The location of the sound in space can play a significant role in detection. To the degree that sounds can be spatially separated in an environment, the potential for masking (i.e., the impact of one sound on the perception of another sound) can be significantly reduced, making the alerts more readily detectable.

Localization. The external flight environment is a spatial one in which events are occurring at different locations in space. Often times, this location information is critical to the pilot and the decisions he or she will ultimately make. In traditional aviation alerting systems, auditory information has not typically had a meaningful directional component. An interesting methodology for providing location information to a pilot is through the use of directional sound, via "spatial auditory displays ${ }^{1}$." With this type of aural display a pilot can perceive "virtual" sounds that can originate from arbitrary azimuth angles, elevations, and distances relative to the listener. Such displays allow ANCOA to take advantage of the natural orienting mechanism of the human auditory system. If an aural alert emanated from the specific location of the threat relative to the pilot of the aircraft (say, for instance, from the direction of another aircraft that is dangerously close), the pilot's response would be to immediately look in that direction, see the threat, and make an evasive maneuver. In this scenario, it has been postulated that the pilot would

\footnotetext{
${ }^{1}$ The use of the term " display" in reference to audition may seem somewhat unnatural; the term is most often associated with the visual display of information. However, a display is simply the "presentation of information to any of the five senses" (Stokes, Wickens, and Kite, 1990, italics added). Therefore, displays can be visual, auditory, tactile, olfactory, or gustatory (taste).
} 
Michael C. Dorneich, Patricia M. Ververs, Michael D. Good (2001). "Design and Evaluation of an Integrated Avionics Alerting System", Proc. of the 20 $0^{\text {th }}$ Digital Avionics Systems Conference. Daytona Beach, FL, 14-18 October 2001

initiate the maneuver more quickly since he or she would not have to localize the other aircraft via visual displays on the flightdeck before reacting. This can be especially important during timecritical situations. This localization, through the aural cue, is a key function of the ANCOA alerting system.

Categorization. There are literally thousands of separate potential conditions that merit an alert. To avoid processing and information overload, and to help pilots to understand the general nature of the alert more quickly, ANCOA defines four high-level categories of "outside-the-aircraft information": traffic, terrain, weather, and an "other" category, which includes information from a variety of sources (e.g., ATC, dispatch, Notices to Airmen NOTAMS). In the ANCOA concept, unique aural and visual representations are used to aid the pilot in differentiating these categories. Providing high level categorization in the aural cue is another innovative aspect of ANCOA.

Prioritization. All failure conditions are not equal. Some conditions may require an immediate response while others may be less critical in nature. Several factors play an important role in determining the criticality of a condition: time to response, certainty of the information, and level of threat to the aircraft and mission. Existing Caition and Warning Systems (CAWS) like EICAS have a prioritization schema: warnings, cautions and advisories. ANCOA, somewhat similarly, has three categories: (1) Time-Critical, (2) Tactical, and (3) Strategic. These categories are roughly equivalent to the warning, caution and advisory categories used in EICAS, but are defined somewhat differently because of the different nature of internal and external conditions.

A time-critical alert is for conditions that demand a response within 60 seconds, When a time-critical situation arises, the crew is presented with a correlated aural/visual alert that is designed to quickly direct their attention to the nature and location of the threat and also to command the pilot on what actions to take to evade the threat. Tactical alerts, on the other hand, have less urgency than time-critical alerts, but still require the pilot's attention to the situation, and have a high probability of requiring pilot response in the near future. These alerts are concerned with problems that may affect the mission within 10 minutes, such as weather in the immediate vicinity, or conditions that may be further away in time but are certain and can be responded to immediately, such as a closed runway or faulty equipment. The philosophy behind strategic alerts for ANCOA is probably the most innovative inclusion to the alerting concept. These alerts address problems that are at least 10 minutes away and those that are probabilistic, such as, a weather cell that is near the destination that is moving away from the airport or pilot reports of wind shear. While this strategic information is important to the overall situation awareness of the crew for planning and informed decisions, the notification system for strategic information must be designed such that the crew does not have their current tasks interrupted, or be overloaded with new information. Therefore, we have developed subtle, yet informative, aural and visual alerts that will allow the crew to decide whether or not they will address the situation then, or at a latter time, depending on their current workload.

Description. Time permitting, a pilot's ability to determine a course of action is greatly enhanced by a deeper understanding of the nature of the condition. Thus a description of the condition can facilitate better decision making by the pilot. In the ANCOA concept, this information can range from a single spoken and visually displayed word indicating traffic in a time-critical situation, for example, to a fairly detailed combination of graphic and textual description of a weather cell over the destination airport for a strategic situation.

Response. A pilot may or may not have time to determine a response to an alert. Depending on the criticality of the situation and the pilot's understanding of the situation, an alerting system may have to provide pilots with direction of the appropriate response to take. This is especially important in time-critical situations where pilots have little time to consider options. In the ANCOA concept, we have included commanded information, both aurally and visually, for these situations. This information takes the form of simple voice commands and visual icons to aid the pilot in rapid decision making. 
Michael C. Dorneich, Patricia M. Ververs, Michael D. Good (2001). "Design and Evaluation of an Integrated Avionics Alerting System", Proc. of the 20 $0^{\text {th }}$ Digital Avionics Systems Conference. Daytona Beach, FL, 14-18 October 2001

\section{Implementation of the ANCOA Concept}

The ANCOA concept is realized in a series of displays, including a directional sound display, a three-button Master-Caution light display, new symbology on the Primary Flight Display (PFD), a message display, and finally, a new dedicated visual display to integrate graphical information from a variety of alerting systems. The following section will describe the properties of these displays and how they support the six functional requirements of ANCOA described in the previous section (functional requirements appear in parentheses).

Directional Sound. Aural alerts are an effective way to get a pilot's attention (Detection). The 3D aspect of the alert gives the pilot directional information (Localization) of either threat and/or response direction during time-critical alerts, or to direct the pilot's attention to the proper display for tactical alerts. The presentation of the alert will differ for the priority associated with the alert (Prioritization). Time critical alerts are presented as voice messages, to better capture the pilot's attention (Detection). The voice message will emanate from the direction of the threat (Localization) and identify the nature of the threat (Categorization). If a command is associated with the time-critical alerts (Response), the command will emanate from the direction of the commanded maneuver (Localization). Tactical alerts will emanate from the MAESA display in order to direct the pilot's attention to the proper visual display (Localization). It will be a non-speech sound (Prioritization) and be one of four distinct sounds one for each category of information (Categorization). Strategic alerts, with four distinct possible non-speech sounds (Categorization), are sounded from the pilot-not-flying's outboard side in order not to distract the pilot-flying's attention if their workload is high (Prioritization).

Master-Caution. In the ANCOA concept, the Master Caution display contains three buttons/lights, one for each priority: time-critical (red), tactical (yellow), and strategic (cyan). When an alert sounds, the appropriate button blinks, to alert the pilot to a condition (Detection, Prioritization). Pressing the button turns the light off and inhibits the aural aspect of the alert.

Primary Flight Display. During timecritical events with a commanded maneuver
(Response), symbology will appear on the primary flight display since that is the most likely place of pilot's attention. For commands that involve a direction (i.e. "PULL UP, PULL UP") an arrow indicates the direction the pilot should fly in response to the threat, where appropriate. The arrow is labeled with the category of the threat (Categorization), and the desired response.

Message Alert and External Situation Awareness (MAESA) Display. One of the most significant elements of the ANCOA concept is the Message Alert and External Situation Awareness (MAESA) Display, illustrated in Figure 3. Timecritical, tactical, and strategic messages and associated graphical representations are displayed visually to the pilot on the MAESA display. This display is situation awareness, planning, and collaborative decision making tool. It allows pilots to view any flight phase and overlay information from multiple sources to gain an understanding of the current and future flight environments. This display consists of two parts: a message area and a graphics area. Each of these components is described in turn.

Message Area. The lower half of the MAESA displays text. The message area can be toggled between two displays via tabs (current messages, and a history log of messages). Textual descriptions of the alert conditions (Description) are displayed on the main tab, ANCOA. The timecritical alerts appear at the top of the message area, followed by the tactical alerts and then the strategic messages at the bottom (Prioritization). Timecritical alerts are displayed in red, tactical alerts are displayed in yellow, and strategic alerts are in cyan (for new messages) or white (for previously alerting information or status messages). Similar to EICAS, new messages of each priority appear at the top of the list. The pilot can scroll through the messages and select a message by clicking on it. Selecting a message links the message to the graphical representation by drawing a box around it in the Graphics area on the upper portion of the display. Only those messages with an associated graphic representation can be selected. Selecting a message automatically adjusts the range of the graphical area such that the associated graphic item will be visible. The History tab will display a time-stamped log of all the messages displayed during the flight. 


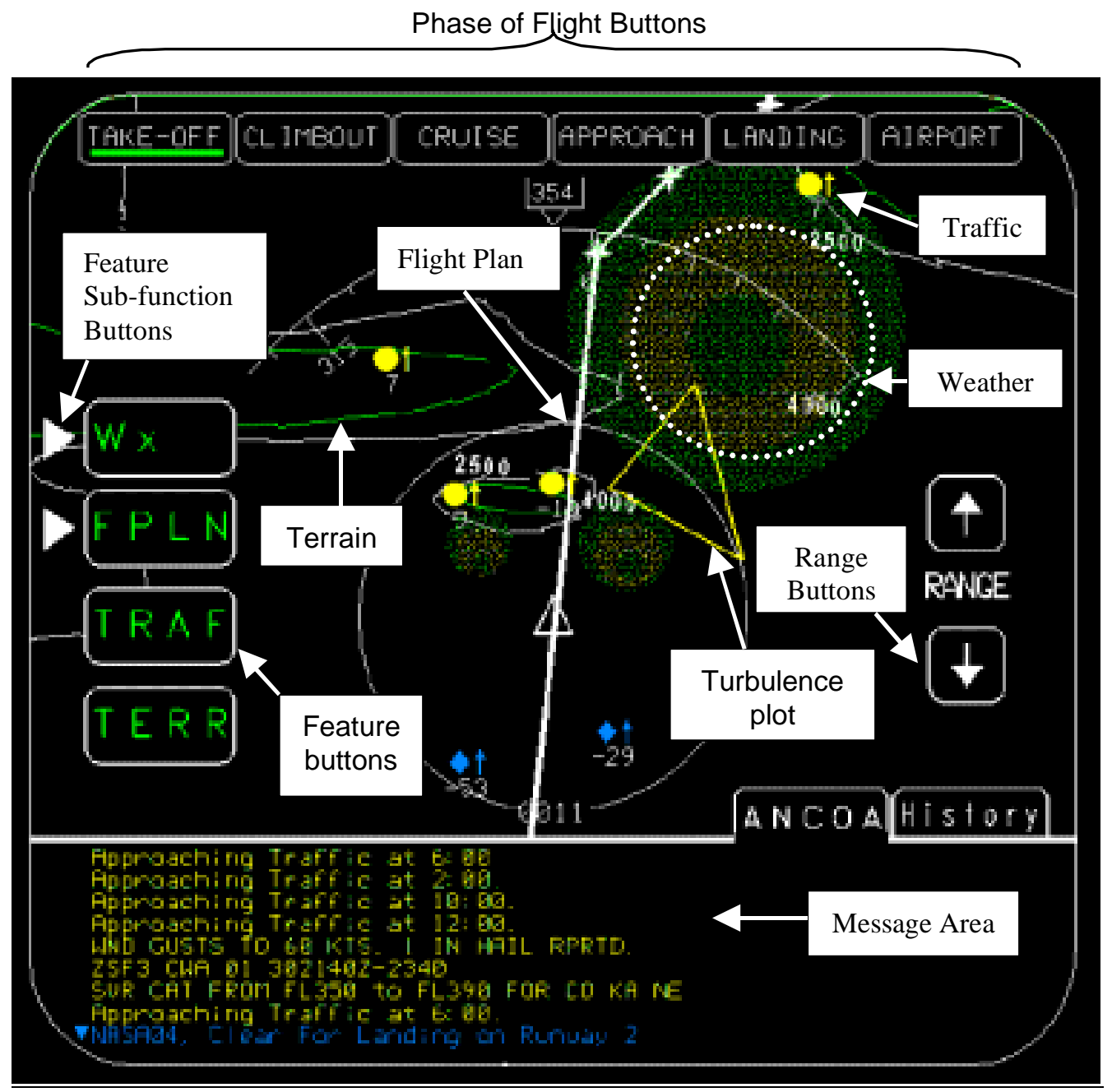

\section{Figure 3. The MAESA Display.}

Graphics Area. The graphics area contains an integrated display of traffic, weather, terrain, and flight plan information. Any of this information can be displayed alone or in conjunction with any other category of information (Categorization). The pilot can select and de-select the information categories depending on the information that he or she will want to use to gain a good sense of the situation.

There are also subcategories of information that are available within each of the key areas. For instance, within weather, the default representation will be the convective weather. However, additional options will allow the presentation of icing, turbulence, winds, wind shear, and volcanic ash. In future implementations, animations could show a time lapse of the weather or traffic in motion, which would allow pilots to acquire some trend information. The flight plan option enables the selection of waypoints, airports, special use airspace (SUA) and runway approaches to be displayed. The traffic button displays the traffic in your general vicinity. Possible terrain sub-function features would enable the pilot to display the topdown, two-dimensional view of the terrain or a rotated three-dimensional perspective display. Other variations include the displaying of absolute terrain features regardless of the pilot's current altitude or terrain represented relative to the current altitude similar to how EGPWS displays terrain.

The second feature of the Graphics area is the phase of flight selection buttons across the top of the display: takeoff, climb, cruise, approach, landing, and airport. When selected, these buttons depict the flight path region that corresponds to the 
Michael C. Dorneich, Patricia M. Ververs, Michael D. Good (2001). "Design and Evaluation of an Integrated Avionics Alerting System", Proc. of the 20 th Digital Avionics Systems Conference. Daytona Beach, FL, 14-18 October 2001

selected flight phase. An additional feature of the display is that multiple consecutive buttons can be displayed. Therefore, the pilot can display the climb, cruise, and approach portions of flight simultaneously. However, non-consecutive portions can not displayed together. Range buttons are provided to fine-tune the region displayed. The airport phase depicts a north-up airport diagram with the runways, taxiways, terminals, and gates. A future feature might include the ability to rotate the map to orient it in the direction of the assigned runway to correspond to track-up position of the final approach. The aircraft's assigned route to the gate is also indicated on the airport map.

There are two views of graphical information the Graphic area. The more common view is an egocentric format, where information is presented in relation to the aircraft's current position along the flight path. Any time the aircraft is in the phase that is selected, an egocentric viewpoint is provided. If the pilot includes consecutive flight phases with the current phase, this egocentric frame is maintained. If the pilot chooses to look ahead in the flight path, beyond his or her current position, an exocentric, world-referenced viewpoint is provided. In the egocentric view, the graphic representation is viewed in a north-up orientation and the display is not referenced to the aircraft's current position. The display options remain the same so that weather, terrain, traffic and flight plan features can be selected and deselected to support situation awareness.

An example of system behavior would be an imminent traffic collision situation, with the threat above the pilot. A speech alert sounds, "TRAFFIC, TRAFFIC", from the direction of the threat, followed by a speech cue "DESCEND, DESCEND" from the direction of the desired response. The aural alert supports detection by capturing the pilot's attention. The priority is timecritical, thus the aural alert is in the form of a speech alert. The location of the threat, and the location of the desired response, is relayed in the 3D locations of the speech alerts. The category of the alert (traffic) is contained in the speech message. Symbology on the PFD (a labeled arrow) will direct a pilot to descend, supporting the response functions (as does the speech cue). The External Situation Display displays an icon (in red) representing the 2D location of the threat relative to the pilot's airplane. The message area contains a message, coded in red as time-critical, which contains further descriptive information.

The prototype ANCOA system described above underwent a usability study, described in the next section.

\section{Usability Study}

Nine pilots participated in six scenarios under various conditions in the Honeywell Laboratories Flight Simulator Laboratory in Minneapolis, MN. This section will discuss the experimental design of the usability study, and summarize the results.

\section{Experimental Design}

\section{Participants}

Nine pilots were recruited for this evaluation. All of the participants were male, ranging in age from 27 years to 62 years, with an average age of 45 years. Seven pilots were from a major airline, one was a commuter pilot and one was a retired airline pilot. Four pilots were currently rated in the DC-9, two pilots were currently rated in the B-757, one was rated in the A-320, and one pilot was rated in the Dornier 328. The retired pilot's last aircraft for which he was rated were the B767/757. Of the active pilots, seven were Captains and one was a First Officer. The total flying time of the pilots ranged from 4,100 hours to 22,000 hours with a mean of 13,300 hours. All but one pilot had experience in glass cockpit aircraft.

\section{The facility}

The ANCOA demonstration was conducted in the Flight Simulation Laboratory of Honeywell Laboratories (HL), a fixed-base, part-task, mediumfidelity simulator. A Silicon Graphics Onyx2 workstation is used to generate the out-the-window (OTW) view of the environment, VAPS visual displays (PFD and MAESA) and the airplane dynamics model, which provides altitude, airspeed, pitch, roll, heading and current position information. The simulator has an OTW display consisting of two $1280 \times 1024$ color monitors located in front of the captain and first officers' seats, two PFDs located below each of the OTWs on flat panel $800 \times 600$ color monitors with a resolution, and the MAESA display on a $1024 \mathrm{x}$ 
Michael C. Dorneich, Patricia M. Ververs, Michael D. Good (2001). "Design and Evaluation of an Integrated Avionics Alerting System", Proc. of the 20 th Digital Avionics Systems Conference. Daytona Beach, FL, 14-18 October 2001

768 touchscreen monitor located between the OTWs and in front of the throttle quadrant. The simulator hardware includes a sidestick, throttle console, and gear lever. The 3-D directional aural signals for the demonstration are generated by a Lake DSP CP4-A4 Digital Audio Convolution Processor with 4 DSPs and a 6-output speaker array. This system is capable of generating multiple sounds that can be presented either statically or dynamically, and allows the simulator to play specific digital sound files and change the spatial location of the alerts in real-time in a threedimensional spatial environment to support the requirements of the ANCOA concept.

\section{Written materials}

A card-sorting task was conducted to gain a better understanding of how pilots group flight information. This was intended to provide us with insight to help determine the appropriate categorization and prioritization for incoming information, such as traffic, icing, winds aloft, temperature, and gate status. The deck of cards contained 59 descriptors of information that could be encountered during a mission. The descriptors were based on a list derived by Riley et al. in an earlier NASA report [4]. Pilots were given instructions to sort the cards into categories based on the similarity of the descriptors, and to provide names for the categories they created. The pilots were also asked to rank the categories in order of importance to them.

A short questionnaire was given after each flight scenario to get the pilots' opinion about specific elements of the ANCOA interface. The pilots were asked to state the extent to which they agreed or disagreed to statements pertaining to the scenario, and were encouraged to give additional comments about the scenario.

A general questionnaire was also created and aimed at the general usability of the ANCOA concept. The questionnaire included questions about preferences for the design and layout of the MAESA display, ease of identifying and locating information, navigation of information, and effectiveness of aural and visual cueing.

\section{Procedure}

Upon arriving at HL, the pilots were briefed as to the purpose of the study and were asked to fill out the consent and demographics forms. They then performed the aforementioned card-sorting task. After completing of the card-sorting task, the subjects were taken to the flight simulator and given an introduction to the ANCOA concept, including an explanation of the MAESA display and threedimensional aural cueing. The pilots completed six scenarios that demonstrated ANCOA's functionality. The order of the scenarios was counterbalanced across the subjects. While performing the scenarios the pilots were asked to think aloud, that is, to describe in words what was going through their mind as they interacted with the prototype during each scenario. If the subjects fell silent for a period of time, they were reminded to keep thinking aloud. After completing each scenario the pilots answered questions from the corresponding post-scenario questionnaire. After completion of all the scenarios each subject completed the general questionnaire and a debriefing interview was conducted. The entire evaluation was videotaped and lasted 2.5 hours.

\section{Results and Discussion}

\section{General Questionnaire Results.}

The results are summarized below including pilot comments from the general usability questionnaire. The scale for numerical responses was 1 (strongly disagree) to 7 (strongly agree).

General Look and Behavior of Concept. The pilots were asked how they liked the "look and behavior" of the concept. An average of 4.4 indicates neither a strong agreement nor disagreement with this question. One pilot noted that the touchscreen was new to him but he liked everything in one location. Another question asked if the "terminology was easy to understand." A 4.8 average suggests moderate agreement with this statement. A couple of pilots mentioned that they reverted to the symbols and commands during the time-critical alerts. They felt that the text messages were informative but too long. In general, when asked about the "effectiveness of the overall conceptual design," the pilots agreed that the design was effective (5.5 rating).

\section{Organization and Functionality. When asked} if they felt ANCOA had all the functions they expected, the pilots moderately agreed with a mean response of 5.1. Since ANCOA is a new concept, the pilots weren't completely sure if ANCOA 
Michael C. Dorneich, Patricia M. Ververs, Michael D. Good (2001). "Design and Evaluation of an Integrated Avionics Alerting System", Proc. of the 20 th Digital Avionics Systems Conference. Daytona Beach, FL, 14-18 October 2001

covers all the functions they might need or want. With a mean response of 4.8 , the pilots slightly agreed that the system navigation was clear and predictable, but they complained that there is no feedback when they went off course. The pilots agreed that related information was grouped logically (5.0 rating) and that it was easy to locate information and functions (4.6 rating). They felt that most of the information they needed was present, and that with experience they could more easily use the touchscreen to get more information. With a mean rating of 5.3, they thought the information on the MAESA screen was easy to understand, but one pilot commented that it wouldn't be necessary to display a text alert for traffic if it was already displayed on the graphics screen. There was strong agreement (6.5 rating) that the response indications on the PFD were useful and intuitive.

Categorization and Prioritization. The pilots felt that it was easy to determine the priority of alerts (5.8 rating), which was aided by the use of different colors for the strategic, tactical, and timecritical priorities. They also agreed that it was usually easy to identify the highest priority alert (5.6 rating), but one pilot said there could be confusion when multiple priority events occurred. With a mean response of 5.6, pilots agreed that they could easily determine the alert category, but one pilot said he just responded to directions given by ANCOA and didn't think about the category of alerts. Finally, the pilots found the directional audio cueing to be ineffective (2.6 rating). Most pilots were unable to perceive sounds originating from different locations within the cockpit. However, upon further investigation, it was determined that the coordinate system used to support the directional audio was not coded properly. This may be one reason that directional audio cues did not provide useful information to the pilots. Further testing is needed to test this concept. Some pilots also expressed concern that 3D audio cueing would be difficult to implement in a cockpit.

\section{Card Sorting Cluster Analysis}

The results of the card-sorting task revealed three main categories of information organized by the pilots. Group A contained weather hazards and information about the environment. Group B contained operational considerations pertaining to navigation and flight constraints. Group $\mathrm{C}$ contained all the items that pilots may consider during a flight, but have little or no impact on the actual flying of the aircraft and safety considerations. A thorough discussion of the card sorting cluster analysis can be found in [5].

\section{Usability Evaluation Conclusions}

Though there were many results of the usability study, for summation purposes, we focus on four key areas of ANCOA's implementation: audio features, visual features, prioritization scheme, and integration philosophy.

Audio. The audio dimension of ANCOA was designed to address both detection and localization of the to-be-alerted-to condition, as well as to provide some information about the categorization and prioritization of the condition. Findings indicated that the audio cueing was quite effective at capturing the pilot's attention and indicating that an alert was present. However, pilots found the directional audio cues intending to represent spatial information about the alerts to be inadequate. A potential explanation for the failure of threedimensional audio to provide effective spatial cueing is that the coordinate system defined for the locations of the physical loudspeakers and the coordinate system defined in software were not the same. Therefore, the resulting ineffectiveness of three-dimensional aural cueing might be a matter of a poor implementation of the concept as opposed to a poor theoretical idea.

As previously mentioned, ANCOA included different sounds to alert the pilot to different categories of conditions. The pilots found that the specific aural alerts that were designed to signify weather, terrain, traffic, and other categories were not intuitive; however, the pilots generally supported the idea of distinct alerts for individual categories. Since there were relatively few categories (four in this instantiation of the ANCOA concept), we believe that the pilots could easily learn to associate the specific characteristics of an aural alert to the particular condition or situation. However, it is critical that the number of categories remain fairly low (e.g., less than 5 or so) in order for the pilot to make a quick association between the aural alert and situation. 
Michael C. Dorneich, Patricia M. Ververs, Michael D. Good (2001). "Design and Evaluation of an Integrated Avionics Alerting System", Proc. of the 20 $0^{\text {th }}$ Digital Avionics Systems Conference. Daytona Beach, FL, 14-18 October 2001

A final question concerns whether the audio cues that were designed for this concept were effective cues for alert prioritization. The ANCOA concept employed a voice alert for time-critical conditions, a repeating non-speech cue for tactical conditions, and a one-time, non-speech cue for strategic conditions. It was not clear from the usability findings whether the scheme was effective for indicating the alert priority to the pilots. Therefore, a further examination of this prioritization scheme based on aural cueing is necessary during the next empirical evaluation.

Visual. The functional integration focused the visual implementation on the categorization, prioritization, condition description, and appropriate response information. The visual medium is good at detailing the threat and providing further information such as its specific location and its severity of the threat. In general, most pilots indicated that the aural cues were better at getting their attention, but the visual cues were better at addressing the threat. The visual implementation consisted of visual alerts presented on the Primary Flight Display (PFD) and the MAESA Display. The pilots found the use of the PFD to display timecritical alert to be very beneficial. They noted that this is where their attention is primarily directed during alert situations and the fact that they received information about the situation on the PFD eliminated the need for them to divert their eyes to other flightdeck display surfaces to assess the current situation. Overall, the subjects liked the directional graphics on the PFD display. Several pilots noted that in addition to the useful visual information that was presented on the PFD (e.g., response information such as 'climb'), additional information such as the amount of input response required to address the situation (e.g., either the rate or ultimate target altitude) would also be very useful. However, one subject disliked the idea of targets because he felt that pilots might focus too much on the PFD and attaining the target and not look out the window or other instruments. If targets were used, he suggested having the arrow size dependent on how much control action is necessary. The pilots also wanted to have some indication on the PFD when the aircraft was clear of the hazard.

Pilots where asked if the would like to see a Vertical Profile Display (VPD) on the MAESA.
Most subjects felt that a vertical profile feature would not be necessary and might even cause information overload. They are interested in vertical profile information but feel that they get this information effectively from the horizontal map through traffic altitude numbers, etc. However, since pilots were asked to assess the idea of a VPD without any concrete representation to react to, the authors feel that the idea merits further study.

The phase of flight categorization on the MAESA display appears to be appropriate. But, some subjects found the phase of flight buttons difficult to work with. Subjects also generally liked the airport tab.

Another component of the visual implementation was the message area on the MAESA where the textual alerts and notifications were integrated, prioritized and displayed. Pilots often found the area became too cluttered with multiple alerts, the messages were too long, and the text was too small. Furthermore, the pilots did not like the strategic information integrated with the time-critical alerts. This may have caused the messages to be difficult to read and understand, potentially resulting in pilots missing important information. Finally, the history tab was not considered useful during flight. Most subjects felt it might only be useful after a flight for writing incident reports after a close call.

As mentioned previously, pilots could select the message and have it highlighted on the graphics display area of the MAESA. Opinions regarding the interaction with the message area were mixed. The pilots generally liked the ability to highlight the individual messages to display its graphical depiction in the graphics area. However, they did note that the graphics area quickly became cluttered. Some of the pilots mentioned that it might be helpful to have the ability to clear out messages once they read them. In the instances that the information was not linked to a graphical depiction (e.g., destination gate change), pilots would like to be able to click on the message to receive more information (e.g., go to the airport schematic to review the new taxi route). In some situations, there was an automatic reconfiguration of the map range in the graphics area. Pilots had divergent opinions regarding this automatic reconfiguration of the display. Some pilots found it 
Michael C. Dorneich, Patricia M. Ververs, Michael D. Good (2001). "Design and Evaluation of an Integrated Avionics Alerting System", Proc. of the 20 $0^{\text {th }}$ Digital Avionics Systems Conference. Daytona Beach, FL, 14-18 October 2001

disconcerting that the map changes range by clicking a message without any further input from the pilots. While other pilots liked that they were not required to make any more inputs in order for the hazardous condition to be displayed. The area of auto reconfiguration of displays is one that is currently receiving significant attention in the human factors community, and we plan to use this body of work to inform design of an appropriate scheme for display reconfiguration for ANCOA.

Priority. Pilots indicated that they liked the strategic, tactical, and time-critical priority classification. They could easily direct their attention to the highest priority items based on the location of the alerts and color of the textual messages. Though there was some disagreement as to the specific time windows for the individual priorities, the pilots generally indicated that the timing would be dependent on the situation and phase of flight. For example, wind shear is considered more critical during takeoff/landing, with traffic and terrain less. Pilots also noted that they wanted more "anticipatory directions" earlier. That is, if the system was aware of an impending condition, the pilots wanted to know what the anticipated response would be if the condition was eventually encountered.

Integration. The pilots were asked to consider the prospect of integrating the ANCOA system with an EICAS/ECAM type system. Their responses varied. Some pilots believed that an integrated system would be too cluttered, whereas others felt that the message areas could be integrated but the graphics area should remain separate. We believe that a less cluttered messaging area with a better layout would make this option appear favorable to the majority of pilots. Pilots also indicated that ANCOA appeared to cover all the functions that they expected to see in such a concept and the information was grouped in a logical manner. They found that the information on the MAESA display was intuitively laid out making it easy to interact with the features. This information suggests that the underlying philosophy and concept implementation scheme has validity. However, it should be noted that more significant testing needs to be done in order to gain a more complete understanding of the utility of the information integration philosophy of the concept.

\section{Revised Prototype and Future Work}

Figure 4 depicts a revised prototype concept for the MAESA display, re-designed with feedback from the usability study. The message area has been moved to the right side and the message length reduced. The pilot can click on the short message to see more detailed information appear directly below the message. To address the readability problems identified, message backgrounds are now colored according to the priority (time-critical $=$ red, tactical = yellow, strategic $=$ cyan , rather than the message text itself. An icon to the left of each message identifies the category of the information. Strategic messages have been moved to a separate tab.

Buttons for each direction have been added to the map display to facilitate navigation. The filter buttons along the left side allow the pilot to choose which information to display on the map display. A vertical profile display (VPD) has been added at the bottom of the display to give pilots a concrete example to assess during the next evaluation. The map display range is determined via the white box in the VPD, adjustable by the zoom buttons.

A new 3D aural display system, the Lake HuronPCI system, with 12 DSPs and an 8-output speaker array has improved spatialization effects to enhance the pilot's ability to localize the timecritical speech alerts. The new system has resulted in much more discernable localized sounds, and should allow a more robust evaluation of the utility of directional aural cues.

Near-term future work will consist of an experimental evaluation of the revised prototype. The study will focus on the evaluating the effectiveness of ANCOA to improve the pilots' situation awareness through 3D audio, visual display improvements, and enhancements to the concept navigability. Long-term future work includes exploring a broadening of the ANCOA concept in order to integrate with alerting systems for conditions inside the aircraft. It is understood that many cases of control upsets involve some combination of adverse conditions both inside and outside the aircraft. For instance, ice accumulation on aircraft wing surfaces or the loss of an engine will reduce an aircraft's flight operating characteristics and significantly decrease the flight crew's ability to operate the aircraft in certain 


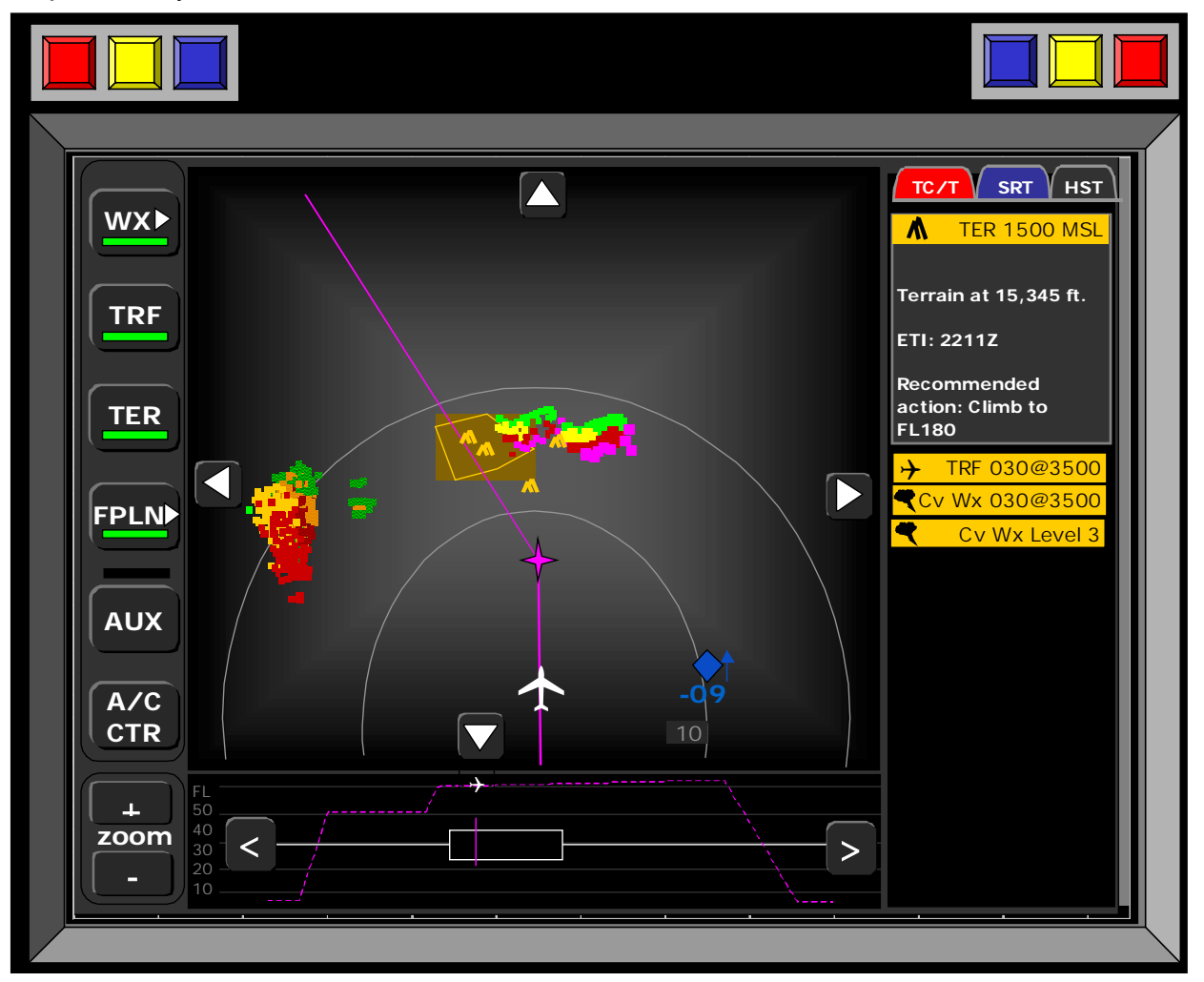

Figure 4. The revised MAESA display.

conditions, such as mountainous regions. The effects of the conditions both inside and outside the aircraft are closely coupled. For this reason, while improvements are being made to the philosophy and conceptual design to ANCOA, the overall framework is being kept open for future internal systems integration.

\section{Acknowledgments}

This research is supported by NASA Langley Research Center: NAS1-20219, Task \#20, Mr. Paul Schutte and Ms. Anna Trujillo, NASA Technical Monitors, and NAS1-00107, Drs. Christine and Celeste Belcastro, NASA Technical Monitor. The authors would also like to thank Dr. Bill Rogers, Jeff Rye, Peter Roeber, Greg Niehus, and Dr.

Wende Dewing for their contributions to this work.

\section{References}

[1] Boucek, G. P., D. C. Hanson, B.L. Berson, M. F. Leffler, D. A. Po-Chedley, and, J. F. Hendrickson, 1981, "Aircraft alerting systems standardization study," AIAA/IEEE Digital Avionics Systems Conference, AIAA: New York, 119-128.

[2] Lindholm, T., 1998, "Cockpit access to strategic weather information," Flight International, 70-74.

[3] Perrott, D. R., T. Sadralodabai, K. Saberi, and T. Z. Strybel, 1991, "Aurally aided visual search in the central visual field: Effects of visual load and visual enhancement of the target," Human Factors, vol. 33, no._4, pp. 389-400.

[4] Riley, V., R. DeMers, M. Good, K. Krishnan, C. Miller, and C. Misiak, 1999, "Crew-Centered Flight Deck Alerting," NASA Technical Report Contract No. NAS1-20219. Hampton, VA: NASA Langley Research Center.

[5] Ververs, Patricia May, Michael C. Dorneich, Michael D. Good, 2001, "Pilot Alerting and Notification of Adverse Conditions - Escape and Avoidance: Achieving PANACEA", to appear in Proceedings of the $45^{\text {th }}$ Annual Meeting of the Human Factors and Ergonomics Society, Santa Monica, CA: Human Factors and Ergonomics Society. 